\title{
President's Corner (Mitteilungen der SGSS)
}

Schmerz 2019 · 33:182

https://doi.org/10.1007/s00482-019-0365-3

(c) Springer Medizin Verlag $\mathrm{GmbH}$, ein Teil

von Springer Nature 2019

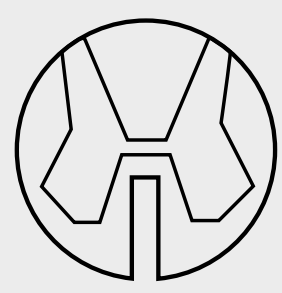

Schweizerische Gesellschaft zum Studium des Schmerzes

Zentralsekretariat SGSS

c/o Pomcanys Marketing AG

Aargauerstrasse 250

8048 Zürich

Tel. +41444961016

E-Mail: info@pain.ch

Web: www.pain.ch

Redaktion

PD Dr. med. Konrad Maurer, Präsident SGSS (v. i.S. d. P.)
Liebe Mitglieder der Schweizerischen Gesellschaft zum Studium des Schmerzes!

Am 1. Februar hat sich der Vorstand der SGSS zu seiner jährlichen, eintägigen Retraite in Bern getroffen.

Verschieden Themenschwerpunkte kamen zur Diskussion, von welchen ich hier die wichtigsten erwähnen will.

\section{Global Year against Pain in the most vulnerable}

Wir wurden von unserer „Mutterorganistion“ IASP aufgefordert, uns als Gesellschaft am diesjährigen „Global Year against Pain in the most vulnerable " $\mathrm{zu}$ beteiligen. Es finden verschiedene Aktivitäten zum Thema statt, welche durch die IASP organisiert werden. Unter Anderem werden Webinars zum Thema abgehalten, welche gratis über die Website der IASP an festgelegten Daten angeboten werden. Unsere Gesellschaft wird sich anlässlich unseres Jahreskongresses am 12ten Internationalen Symposium der Pädiatrischen Schmerzen am 16. Juni 2019 in Basel mit einem Stand der SGSS beteiligen, und weitere Informationen zum Thema auflegen können.

\section{Mitgliederbeiträge}

Ein weiteres Thema sind die Mitgliederbeiträge. Wir haben einen Vorschlag erarbeitet, die Beiträge etwas $\mathrm{zu}$ diversifizieren. Unsere Gesellschaft ist wie jedes Kapitel der IASP eine multidisziplinäre Gesellschaft und wir sind der Meinung, dass der Mitglieder- beitrag für die verschiedenen Berufsgruppen entsprechend der finanziellen ,Kräftigkeit ' Rechnung getragen werden sollte. Entsprechend sehen wir vor, für die einen den Mitgliederbeitrag um $10 \%$ $\mathrm{zu}$ erhöhen und für die anderen etwas zu reduzieren. Unter dem Strich gibt es nach dem erarbeiteten Modell eine Zunahme der Einnahmen für die Gesellschaft um ca. $4 \%$. Die Generalversammlung im Juni wird über diesen Beschluss das letzte Wort haben.

\section{Vereinfachung der Prozesse}

Weiter planen wir eine Vereinfachung der Prozesse der Aufnahme von neuen Mitgliedern in die Gesellschaft. Dank der immensen Arbeit unseres Vorstandmitgliedes Beat Steiger konnte innerhalb des letzten Jahres eine moderne Mitgliederdatenbank erstellt werden, welche uns erlaubt, in Zukunft viel speditiver und viel gezielter Informationen unserer Gesellschaft zu analysieren. Ebenfalls wird das Verschicken von Mitteilungen elektronische Newsletter oder der Prozess der Rezertifizierung des Titels „Schmerzspezialist SGSS“ dadurch viel einfacher und effizienter. Ich möchte mich an dieser Stelle im Namen der SGSS ganz herzlich bei Beat bedanken!

\section{Namensänderung}

Als letzten Punkt möchte ich die Diskussion bezüglich des $\mathrm{Na}$ mens der Gesellschaft lancieren. Die meisten Kapitel der IASP haben einen eingängigen und einfachen Namen (z. B. British Pain Society BPS, Deutsche Schmerz- gesellschaft DSG etc.). Wir vom Vorstand sind der Meinung, dass auch unsere Gesellschaft eine kürzere Bezeichnung braucht und uns diesbezüglich anpassen sollten. In der Schweiz ist das natürlich nicht so einfach wie in unseren Nachbarländern, weil wir verschiedene Landessprachen haben. $\mathrm{Zu}$ allem Überfluss erscheint in unserem aktuellen Logo auch noch eine englische Version des Namens. Unser Vorstand schlägt deshalb vor, unsere Gesellschaft in Zukunft schlicht „Swiss Pain Society“ zu nennen, wie das zum Beispiel in Belgien, welches auch mehr als eine offizielle Landessprache besitzt, der Fall ist. Einen entsprechenden Vorschlag werden wir der nächs- ten Generalversammlung zur Abstimmung präsentieren und sind auf die sicherlich lebhafte Diskussion darüber gespannt.

Es bleibt mir nur noch auf die nächsten wichtigen Veranstaltungen der SGSS/SSED/ASSD/ SASP (in Zukunft vielleicht nur noch SPS) hinzuweisen und freue mich, Euch bald an diesem oder jenen Event persönlich zu treffen!

Beste Grüsse!

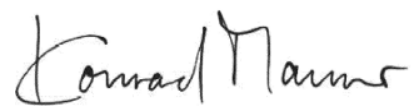

PD Dr. med. Konrad Maurer SGSS Präsident

\section{Wichtige Events 2019}

(Detaillierte Informationen zu jeder Veranstaltung finden Sie auf unserer Website www.pain.ch)

\section{SGSS Schmerzkurs 2019}

\section{"Schmerztherapie nach}

Schmerzmechanismus"

Der multidisziplinärer Schmerzkurs findet in 5 Abschnitten von März bis Juni 2019 im Sorell Hotel Zürichberg in Zürich statt.

\section{ACHD Dreiländertagung}

Am 29. und 30. März 2019 möchten wir uns mit der deutschen und österreichischen Schmerzgesellschaft in Wien treffen.

\section{SGSS Jahreskongress 2019}

Am Education Day des 12ten Internationalen Symposium der Pädiatrischen Schmerzen, am Sonntag, 16. Juni 2019 in Basel, findet unser Jahrestreffen statt. Ein interessantes Programm mit Sprechern aus der ganzen Welt erwartet sie.

\section{SSED Cours La douleur 2019}

"LA DOULEUR, des mécanismes aux traitements"

Le cours multidisciplinaire sur la douleur se déroule en $3 \mathrm{sec}$ tions d'octobre à décembre 2019 à Morges, Centre de congrès La Longeraie 